\title{
Migraine and Its Connections in Neurology and Psychiatry
}

\author{
By Jack M. Gorman, MD
}

At the recent meeting in Hawaii of the American Academy of Neurology, we heard many positive comments about CNS Spectrums. We were also urged by the attending neurologists to try and include more articles and issues devoted to neurologic disease and mechanisms.

Previously, we have had issues on epilepsy and stroke, and future issues are planned that will cover movement disorders, multiple sclerosis, child neurology, and Alzheimer's disease. We very much want and welcome both suggestions for neurological topics to which future issues may be dedicated and submission of unsolicited original research and review articles dealing with neurological topics. Our peer review process is quick and efficient. Accepted articles will be scheduled to appear as rapidly as possible.

This issue, whose articles I will discuss out of the printed order, is devoted largely to a topic of interest to neurologists and psychiatrists alike-migraine. Four papers deal with various aspects of migraine headache, a common and often plaguing disorder that still mystifies scientists and often eludes response to therapy. It is not an easy condition to diagnose; unlike many neurologic disorders, but in common with most psychiatric illness, there are no definitive objective tests that can make a positive diagnosis of migraine headache. Rather, after ruling out other causes of headache, like brain tumor, the neurologist must rely largely on history. Sometimes, the elicitation of symptoms like an aura can be nearly pathognomonic, but more often migraine is a diagnosis of exclusion by history alone. Treatment relies on palliative measures, including a variety of analgesics, some of which have abuse potential, ergotamine, $\beta$-blockers, anticonvulsants, and serotoninenhancing drugs. Antidepressants generally are not effective in treating migraine headache; in some patients they seem to help while in others they worsen the situation.

Adding to this complexity, as Nancy C.P. Low, MD, MS, and Kathleen Ries Merikangas, $\mathrm{PhD}$, of the National Institute of Mental Health, is the fact that migraine is highly comorbid with a host of other medical problems. These involve almost all organ systems and include psychiatric illness. Unfortunately, this penchant for co-occurrence with other disorders has not shed much light on the fundamental pathophysiology of migraine headache, but it does sometimes obscure the diagnosis. Their article is important as a reminder to think about migraine in palients with headache even in the face of multiple other problems.

Two articles suggest possible new treatment approaches to migraine. Keith R. Edwards, MD, from the Neurological Research Center in Bennington, Vermont, and colleages, from various other locations, combine data from two single-site, double-blind, placebo-controlled trials of the anticonvulsant topiramate for migraine prophylaxis. Seventy patients were enrolled and the results were positive: overall, topiramate produced a lower 28-day migraine frequency than placebo. About five times as many patients responded to topiramate than to placebo, although the overall response rate to the active drug was low (35.3\%). Topiramate-associated adverse events included paresthesia and memory impairment. As has been observed before, topiramate also caused appetite suppression and weight loss. As I am a consultant to the manufacturer of topiramate (and therefore did not participate in the review of this article), I will refrain here from making any conclusions except to say that future studies of topiramate will be of great interest.

Nabih M. Ramadan, MD, from the Chicago Medical School argues convincingly in his paper that migraine headache may involve an abnormal activation of glutamatergic neuronal pathways and suggests that glutamate antagonists, which appear to work in animal models of migraine, might be useful therapeutically. This work provides both a mechanistic hypothesis and a suggestion for treatment approaches and deserves careful scrutiny by both investigators and clinicians.

By contrast to these positive notes about therapeutics, William B. Young, MD, and colleagues from the Thomas Jefferson University Hospital provide data from a study of 50 patients with severe headache, most of who had migraine, who were admitted to an outpatient infusion center. Seventeen of these patients also had restless legs syndrome and these individuals turned out to have a very high risk of developing akathisia following administration of intravenous dopamine receptor blocking agents. The authors suggest that increased surveillance for restless legs syndrome in headache patients might be important prior to initiating therapy with dopamine receptor antagonists.

A final paper in this issue deals with a completely separate topic, obsessive-compulsive disorder (OCD). Donatella Marazziti, MD, of the University of Pisa and an associate international editor of CNS Spectrums notes that about onethird of patients with OCD do not respond to serotonin reuptake inhibitor medications. Dr. Marazziti reports a positive experience with venlafaxine for such patients, a medication that blocks both serotonin and norepinephrine reuptake in a manner similar to clomipramine but with fewer adverse events. This is welcome news for clinicians and patients alike dealing with the often very hard to treat syndrome of OCD.

Finally, I want to remind our readers that CNS Spectrums is now accepting letters to the editor. Please send them in! CNS 


\section{A different path to success in your continuing treatment of schizophrenia}
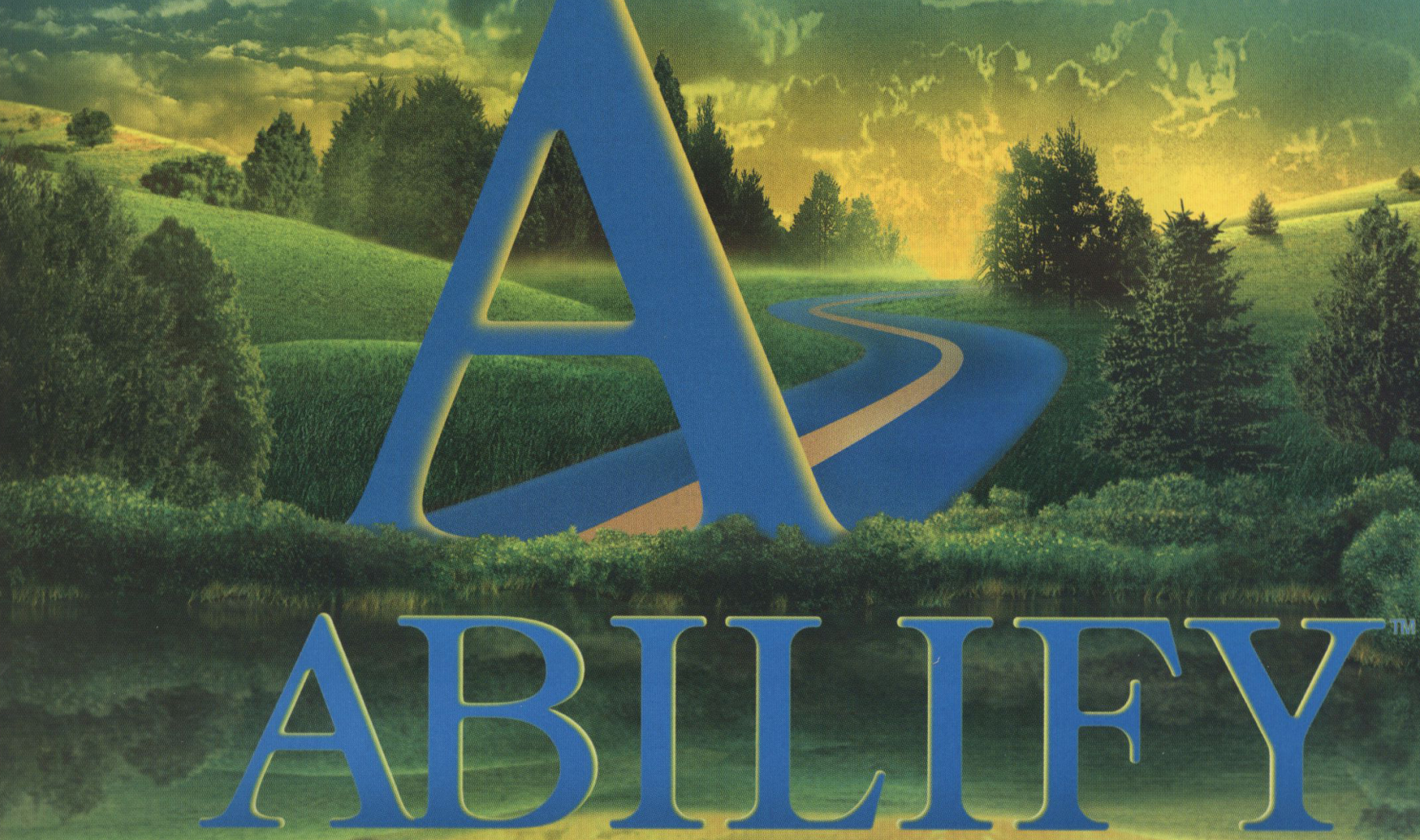

- (aripiprazole)

DISCOVER PROVEN EFFICACY, TOLERABILITY, AND SAFETY FOR THE ROAD AHEAD 
road not traveled before

\title{
Unique Pharmacology Sets Abilify Apart ${ }^{1}$
}

\section{Abilify is a partial agonist that uniquely modulates dopamine activity. ${ }^{1,2}$}

- Functional antagonist activity at $\mathrm{D}_{2}$ receptors in a hyperdopaminergic environment ${ }^{1}$

- Functional agonist activity at $\mathrm{D}_{2}$ receptors in a bypodopaminergic environment ${ }^{1}$

Serotonin antagonist activity at $5-\mathrm{HT}_{2 \mathrm{~A}}$ receptors and partial agonist activity at $5-\mathrm{HT}_{1 \mathrm{~A}}$ receptors

Abilify has moderate affinity for alpha ${ }_{1}$-adrenergic and histamine $\left(\mathrm{H}_{1}\right)$ receptors

\begin{abstract}
Abilify has no appreciable affinity for cholinergic muscarinic receptors
\end{abstract}

The mechanism of action of Abilify, as with other drugs having efficacy in schizophrenia, is unknown.

Abilify is indicated for the treatment of schizophrenia.

Please see Brief Summary of Prescribing Information on last page of this insert. 
Effect of Abilify on weight, long term

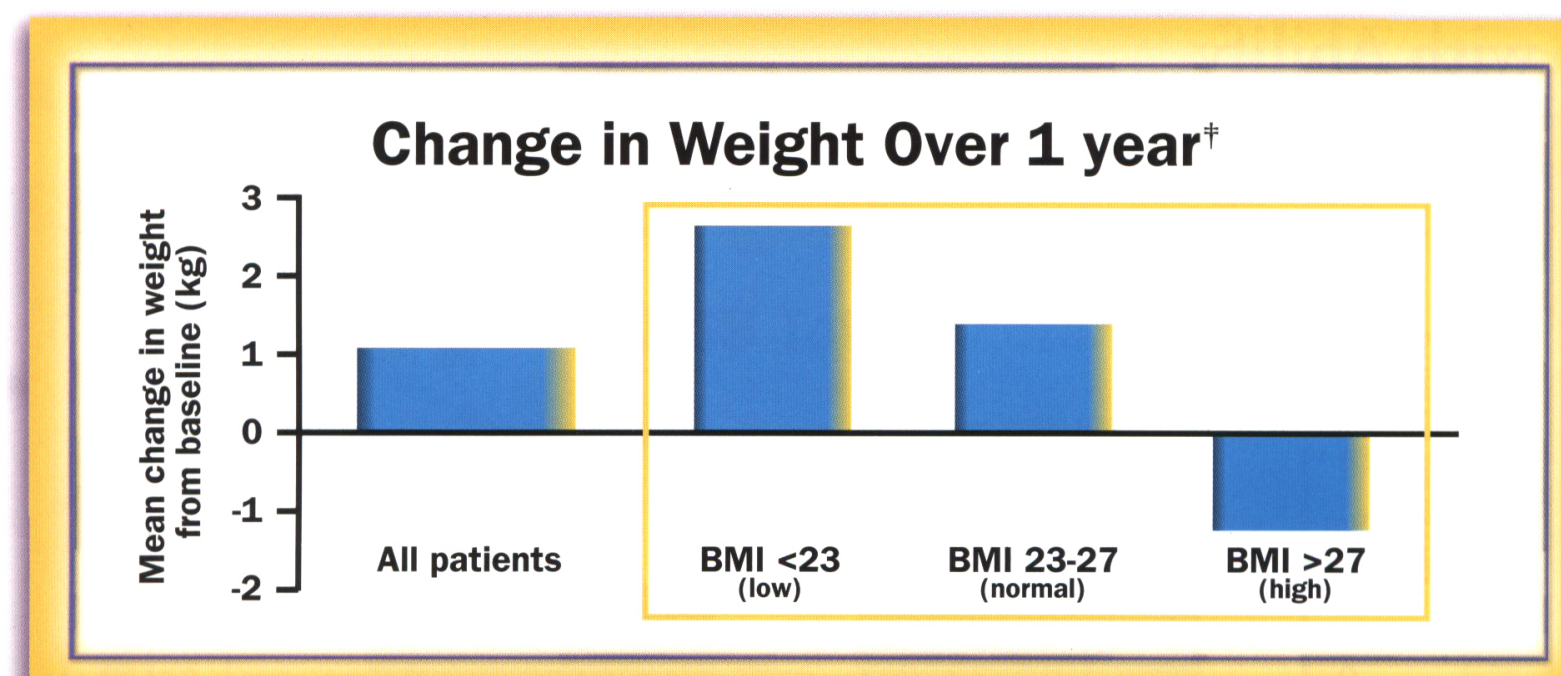

A prospective 52-week, double-blind trial. For Abilify, BMI <23 ( $n=314$ ); BMI 23 to 27 ( $n=265)$; and BMI $>27$ ( $n=260$ ).

The percentage of patients with $\geq 7 \%$ increase in body weight was $30 \%$ for those with $\mathrm{BMI}<23,19 \%$ for those with BMI 23 to 27 ,

and $8 \%$ for those with $\mathrm{BMI}>27$.

†Last observation carried forward

- Because patients' overall health is important

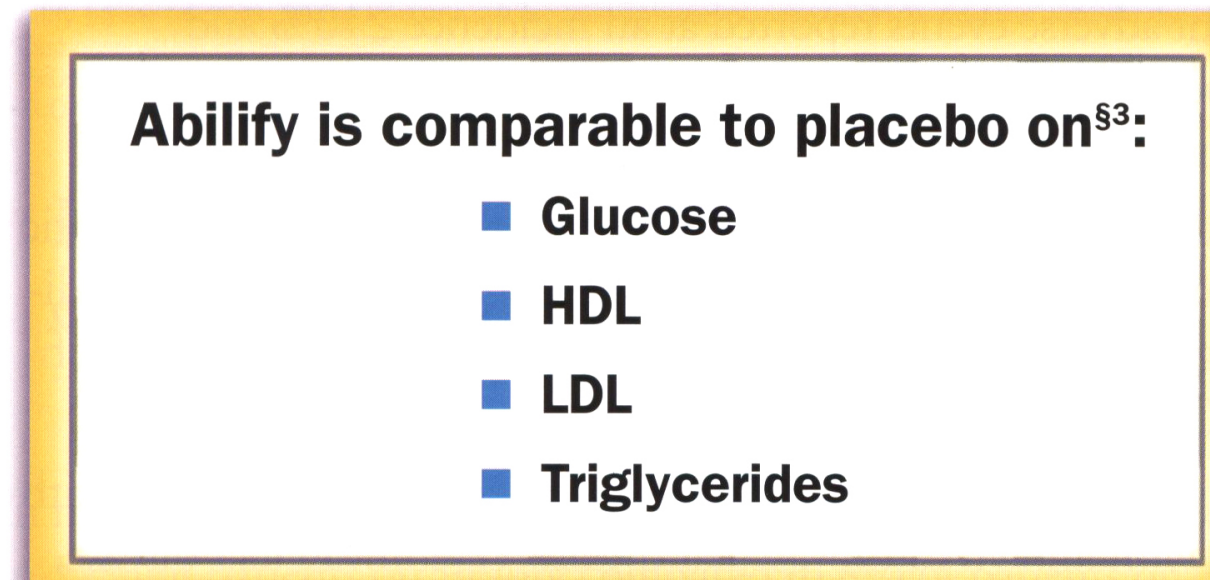

Data from a 6-week, placebo-controlled, clinical trial.

$\S$ As measured by routine serum chemistry analysis.

Please see Brief Summary of Prescribing Information on last page of this insert.

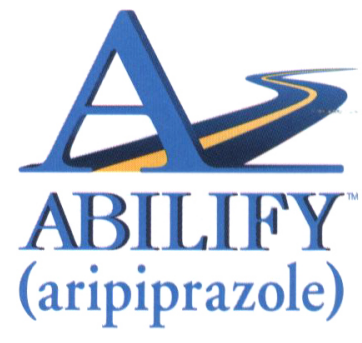


A clear path for the journey ahead

\section{Few patients discontinue due to adverse events with Abilify}

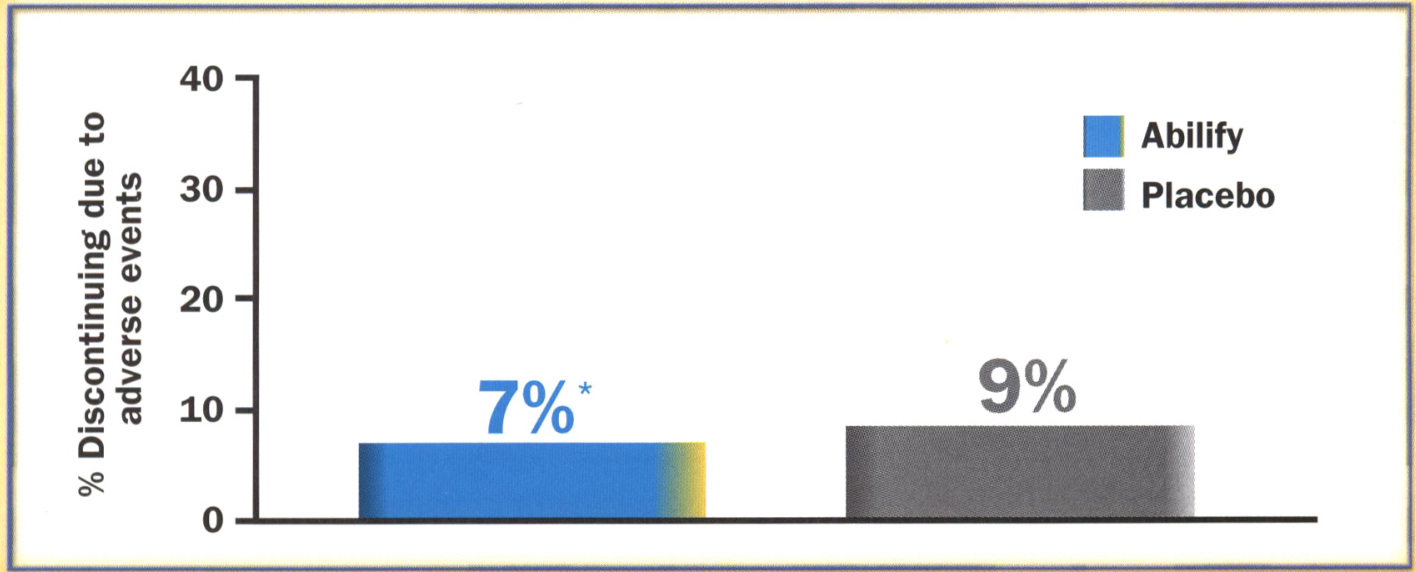

Pooled data from five 4- to 6-week, placebo-controlled clinical trials.

*There is no statistical difference in the incidence of discontinuation due to adverse events, and the types of adverse events

that led to discontinuation were similar between placebo-treated patients and patients treated with Abilify.

Treatment-emergent adverse events reported at an incidence $\geq 10 \%$ and greater than placebo include headache, anxiety, insomnia, nausea, vomiting, lightheadedness, somnolence, akathisia, and constipation.

As with all antipsychotic medications, a rare condition referred to as neuroleptic malignant syndrome (NMS) has been reported. As with all antipsychotic medications, prescribing should be consistent with the need to minimize the risk of tardive dyskinesia (TD).

Abilify may be associated with orthostatic hypotension and should be used with caution in patients with known cardiovascular disease, cerebrovascular disease, or conditions which would predispose them to hypotension.

As with other antipsychotic drugs, Abilify should be used with caution in patients with a history of seizures or with conditions that lower the seizure threshold. Seizures occurred in $0.1 \%$ of Abilify-treated patients in placebocontrolled trials. 


\section{The Confidence of Proven Efficacy}

\section{Significant improvement as early as Week $1^{3}$}

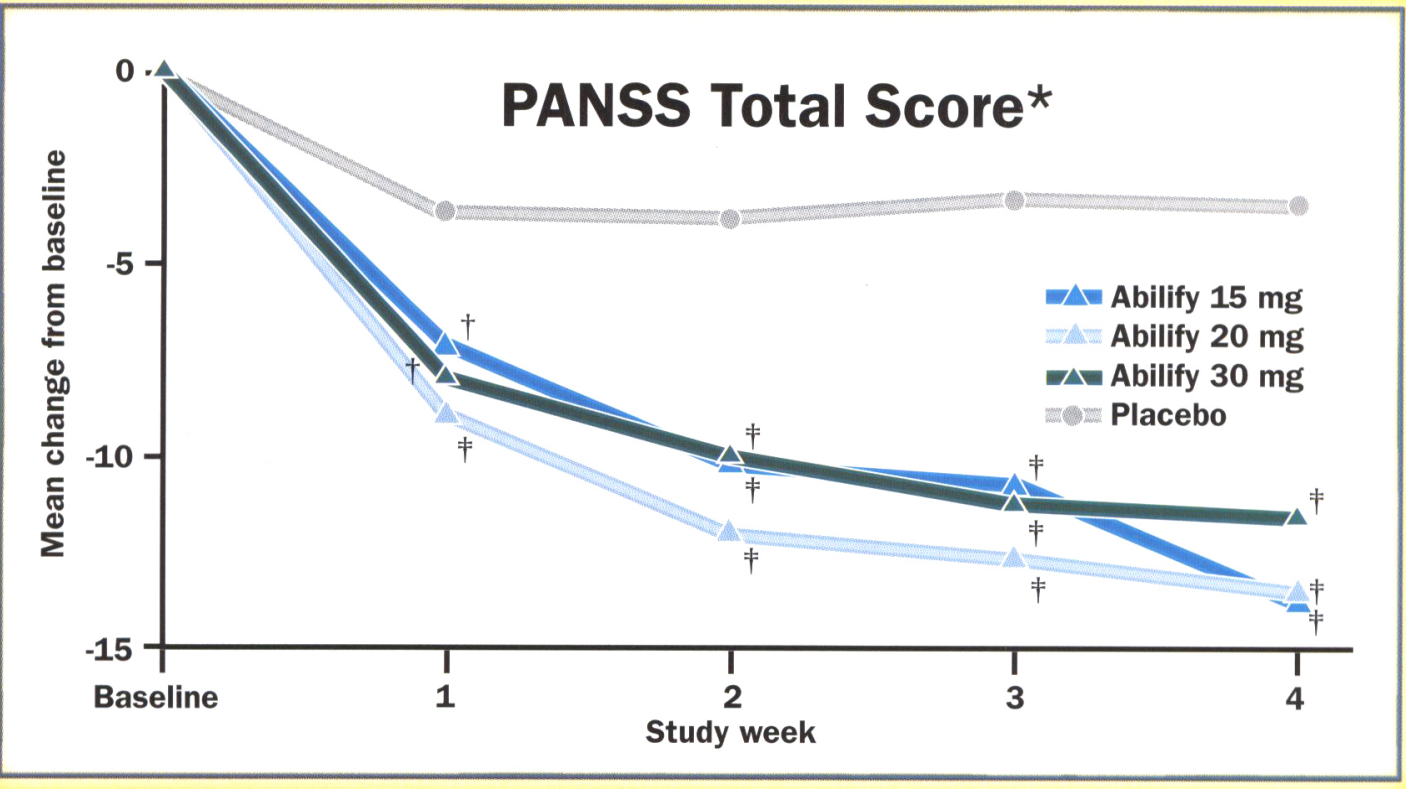

Abilify $15 \mathrm{mg}$ ( $n=202), 20 \mathrm{mg}(\mathrm{n}=195), 30 \mathrm{mg}(\mathrm{n}=196)$, and placebo $(\mathrm{n}=312)$. Analysis included data from all fixed-dose trials.

* Last observation carried forward.

$\dagger P<0.05$ vs placebo.

$\neq P<0.01$ vs placebo.

\section{In efficacy studies, $88 \%$ of responders did not experience sedation ${ }^{3}$}

In multiple, placebo-controlled trials, somnolence was reported in $11 \%$ of patients on Abilify compared to $8 \%$ of patients on placebo; somnolence led to discontinuation in $0.1 \%$ of patients on Abilify in these clinical trials. In clinical trials, the only adverse event to have a possible dose-response relationship was somnolence (placebo, 7.7\%; $15 \mathrm{mg}, 8.7 \% ; 20 \mathrm{mg}, 7.5 \%$; and $30 \mathrm{mg}, 15.3 \%)$.

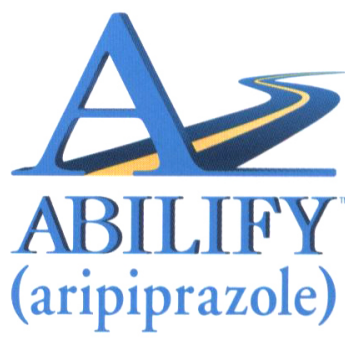


course with few detours

\section{Tolerability and Safety for the Road Ahead}

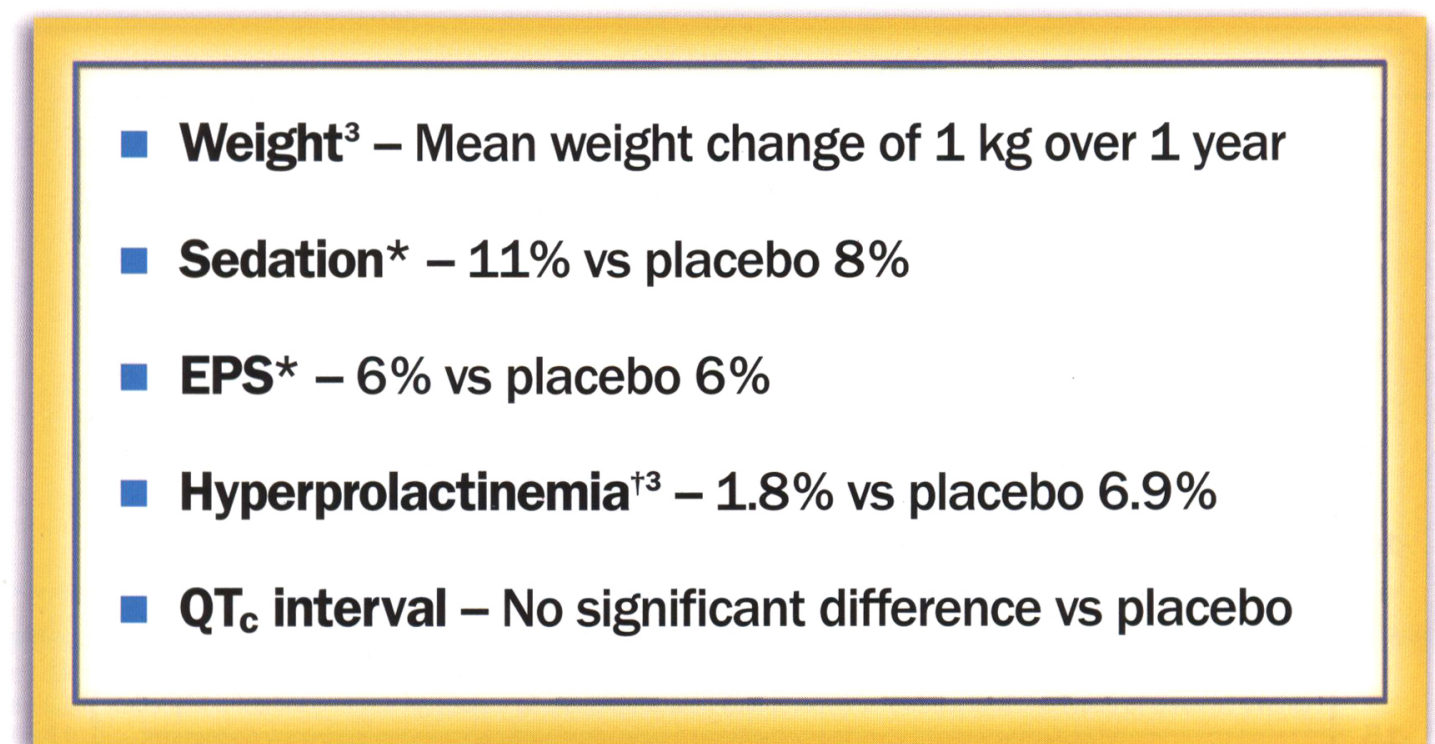

*Patient-reported adverse events in 4- and 6-week placebo-controlled trials.

†In patients with prolactin levels less than or equal to the upper limit of normal at baseline.

In a 52-week study, the percentage of patients with $\geq 7 \%$ increase in body weight was 30\% for those with BMI (Body Mass Index $\left[\mathrm{kg} / \mathrm{m}^{2}\right]$ ) $<23,19 \%$ for those with BMI 23 to 27, and 8\% for those with BMI $>27$.

In short-term trials, there was a slight difference in mean weight gain between Abilify and placebo patients $(+0.7 \mathrm{~kg}$ vs $-0.05 \mathrm{~kg}$ respectively), and also a difference in the proportion of patients meeting a weight gain criterion of $\geq 7 \%$ of body weight for Abilify (8\%) compared to placebo (3\%). 


\section{Abilify Makes Dosing Easy for the Patient and You}
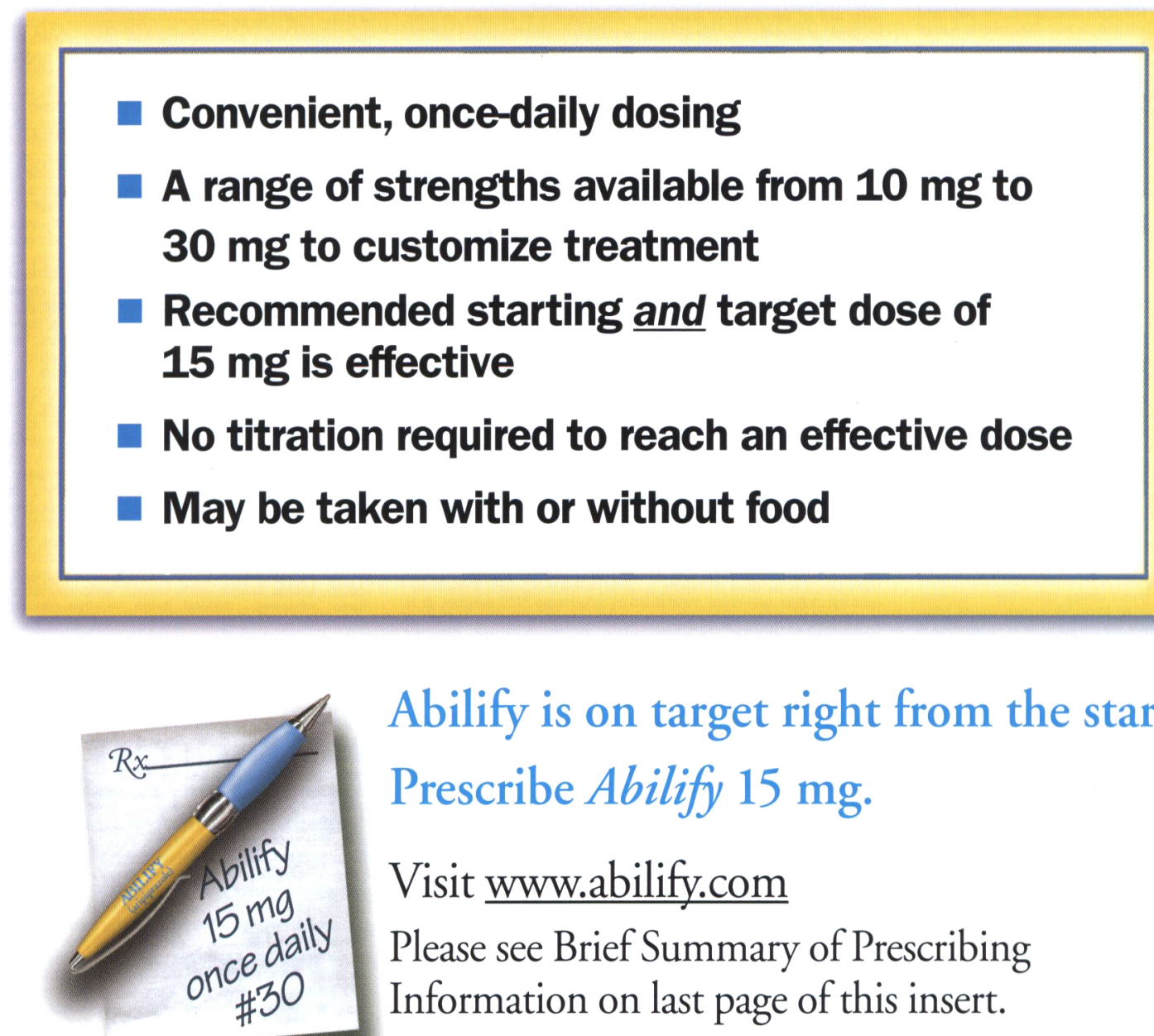

Abilify is on target right from the start. Prescribe Abilify $15 \mathrm{mg}$.

Visit www.abilify.com

Please see Brief Summary of Prescribing Information on last page of this insert.

Retrimese

1. Burris KD, Molski TF, XU C, et al. Aripiprazole, a novel antipsychotic, is a high-affinity partial agonist at human dopamine $\mathrm{D}_{2}$ receptors. The Journal of Pharmacology and Experimental Therapeutics. 2002;302:381-389.

2. Kikuchi T, Tottori K, Üwahodo Y, et al. 7-\{4-[4-(2,3-dichlorophenyl)-1-piperazinyl]butyloxy\}-3,4-dihydro-2

$(1 \mathrm{H})$-quinolinone (OPC-14597), a new putative antipsychotic drug with both presynaptic dopamine autoreceptor agonist activity and postsynaptic $\mathrm{D}_{2}$ receptor antagonistic activity. The Journal of Pharmacology and Experimental Therapeutics. 1995;274:329-336.

3. Data on file. Otsuka America Pharmaceutical, Inc., Rockville, Md.

Marketed by Otsuka America Pharmaceutical, Inc., Rockville, MD 20850 USA and Bristol-Myers Squibb Co., Princeton, NJ 08543 USA.

Manufactured by Otsuka Pharmaceutical Co., Ltd., Tokyo, 101-8535 Japan.

Distributed by Bristol-Myers Squibb Co., Princeton, NJ 08543.

U.S. Patent Nos. 4,734,416 and 5,006,528. 
ABILIFY

\section{(aripiprazole) Tablets}

Brief Summary of Prescribing Information. For complete prescribing information INDICATIONS AND USAGE

ABILIFY (aripiprazole) is indicated for the treatment of schizophrenia. The term (4-and 6-week) controlled trials of schizophrenic inpatients (see CLINICAL PHARMACOLOGY: Clinical Studies). The long-term efficacy of aripiprazole in
the treatment of schizophrenia has not been established. The physician who the treatment of schizophrenia has not been established. The physician who
elects to use ABILIFY for extended periods should periodically re-evaluate the CONTRAINDICATIONS

\section{ABILIFY is CONI}

Neuroleptic Malignant Syndrome (MMS): A potentially fatal symptom complex sometimes referred to as Neuroleptic Malignant Syndrome (NMS) has been reported in association with administration of antipsychotic drugs, including
aripiprazole. Two possible cases of NMS occurred during aripiprazole treatment in the premarketing worldwide clinical database. Clinical manifestations of NMS are hyperpyrexia, muscle rigidity, altered mental status, and evidence of autocardiac dysrthythmia). Additional signs may include elevated creatine phosphokinase, myoglobinuria (rhabdomyolysis), and acute renal failure. The diagnostic evaluation of patients with this syndrome is complicated. In arriving at a diagnosis, it is important to exclude cases where the clinical presentation includes
both serious medical illness (e.g., pneumonia, systemic infection, etc) and both serious medical illness (e.g., pneumonia, systemic infection, etc) and
untreated or inadequately treated extrapyramidal signs and symptoms (EPS) untreated or inadequately treated extrapyramidal signs and symptoms (EPS)
Other important considerations in the differential diagnosis include central antiOther important considerations in the differential diagnosis include central anti-
cholinergic toxicity, heat stroke, drug fever, and primary central nervous system pathology. The management of NMS should include: 1) immediate discontinua-
tion of antipsychotic drugs and other drugs not essential to concurrent therapy 2) intensive symptomatic treatment and medical monitoring; and 3) treatment
any concomitant serious medical problems for which specific treatments ar any concomitant serious medical problems for which specific treatments ar ment regimens for uncomplicated NMS. If a patient requires antipsychotic drug
treatment after recovery from NMS, the potential reintroduction of drug therapy treatment after recovery from NMS, the potential reintroduction of drug therapy
should be carefully considered. The patient should be carefully monitored, since should be carefully considered. The patient should be carefully monitored, since
recurrences of NMS have been reported. Tardive Dyskinesia: A syndrome of potentially irreversible, involuntary dyskinetic movements may develop in patentially irreversible, involuntary, dyskinetic movements may develop in drome appears to be highest among the elderly, especially elderly women, it is antipsychotic treatment, which patients are likely to develop the syndrome. Whether antipsychotic drug products differ in their potential to cause tardive hood that it will become irreversible are believed to increase as the duration treatment and the total cumulative dose of antipsychotic drugs administered to the patient increase. However, the syndrome can develop, although much less commonly, after relatively brief treatment periods at low doses. There is no known treatment for established cases of tardive dyskinesia, although the syn-
drome may remit, partially or completely, if antipsychotic treatment is withdrome may remit, partially or completely, if antipsychotic treatment is with-
drawn. Antipsychotic treatment, itself, however, may suppress (or partially sup-
press) the signs and symptoms of the syndrome and, thereby, may possibly mask the underlying process. The effect that symptomatic suppression has upon the long-term course of the syndrome is unknown. Given these considerations,
ABILIFY should be prescribed in a manner that is most likely to minimize the ally be reserved for patients who suffer from a chronic illness that (1) is known to respond to antipsychotic drugs, and (2) for whom alternative, equally effective, but potentially less harmful treatments are not available or appropriate. In patients who do require chronic treatment, the smallest dose and the shortes patients who do require chronic treatment, the smallest dose and the shortest sought. The need for continued treatment should be reassessed periodically. I
signs and symptoms of tardive dyskinesia appear in a patient on ABILIFY, drug discontinuation should be considered. However, some patie
treatment with ABILIFY despite the presence of the syndrome.

\section{PRECAUTIONS}

General: Orthostatic Hypotension: Aripiprazole may be associated with orthostadence of orthostatic hypotension associated events from five short-term, placedence of orthostatic hypotension associated events from five short-term, place-
bo-controlled trials in schizophrenia ( $n=926$ ) on ABILIFY (aripiprazole) included orthostatic hypotension (placebo $1 \%$, aripiprazole $1.9 \%$ ); orthostatic lighthead
edness (placebo $1 \%$, aripiprazole $0.9 \%$, and syncope (placebo $1 \%$, aripiprazole edness (placebo $1 \%$, aripiprazole $0.9 \%$, and syncope (placebo $1 \%$, aripiprazole
$0.6 \%$. The incidence of a significant orthostatic change in blood pressure (defined as a decrease of at least $30 \mathrm{mmHH}$ in systolic blood pressure when changing from a supine to standing position) for aripiprazole was not statistical-
ly different from placebo (14\% among aripiprazole-treated patients and $12 \%$ among placebo-treated patients). Aripiprazole should be used with caution in patients with known cardiovascular disease (history of myocardial infarction or chemic heart disease, heart failure or conduction abnormalittes), cerebrovas(dehydration, hypovolemia, and treatment with antihypertensive medications) Seizure: Seizures occurred in $0.1 \%$ (1/926) of aripiprazole-treated patients in
short-term, placebo-controlled trials. As with other antipsychotic drugs, aripiprashort-term, placebo-controlled trials. As with other antipsychotic drugs, aripiprazole should be used cautiously in patients with a history of seizures or with con-
ditions that lower the seizure threshold e.g. Alzheimer's dementia. Condition that lower the seizure threshold may be more prevalent in a population of placebo-controlled trials, somnolence was reported in $11 \%$ of patients on ABILIFY compared to $8 \%$ of patients on placebo; somnolence led to discontrole
ation in $0.1 \%(1 / 926)$ of patients on ABILIFY in short-term, placebo-controlled pared to placebo, ABILIFY, like other antipsychotics, may have the potential to impair judgment, thinking, or motor skills. Patients should be cautioned about impair judgment, thinking, or motor skills. Pattents should be cautioned about
operating hazardous machinery, including automobiles, until they are reasonably operating hazardous machinery, including automobiles, until they are reasonably
certain that therapy with ABILIFY does not affect them adversely. Body Temperature Regulation: Disruption of the body's ability to reduce core body advised when prescribing aripiprazole for patients who will be experiencing conditions which may contribute to an elevation in core body temperature, e.g. ication with anticholinergic activity, or being subject to dehydration. Esophageal dysmotlity and aspiration have been associated with antipsychotic drug use. Aspiration pneumonia is a common cause of morbidity and mortality
in elderly patients, in particular those with advanced Alzheimer's dementia. Aripiprazole and other antipsychotic drugs should be used cautiousiy in patients at risk for aspiration pneumonia (See PRECAUTIONS: Use in Patients with Concomitant Illness ). Suicide: The possibility of a suicide attempt is inherent in
psychotic illnesses, and close supervision of high-risk patients should accompapsychotic illnesses, and close supervision of high-risk patients should accompa-
ny drug therapy. Prescriptions for ABILIFY should be written for the smallest quantity of tablets consistent with good patient management in order to reduce in

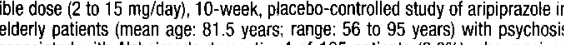
ABILIFY died compared in sementia, 4 of 105 patients $(3.8 \%)$ who receive during or within 30 days after termination of the double-blind portion of the study. Three of the patients (age 92,91 and 87 years) died following the scontinuation of ABILIFY in the double-blind phase of the study (causes of

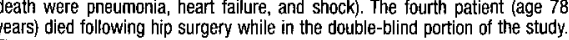
The treatment-emergent adverse events that were reported at an incidence of
$>5 \%$ and having a greater incidence than placebo in this study were accidental jury, somnolence, and bronchis. Eight percent of the ABILIFY-treated patients reported somnolence compared to one percent of placebo patients. In a small
pilot, open-label, ascending-dose cohort study $(n=30)$ in elderly patients with pementia, ABILIFY was associated in a dose-related fashion with somnolence.
The safety and efficacy of ABILIFY in the treatment of patients with psychosis The safety and efficacy of ABILIFY in the treatment of patients with psychosis
associated with dementia have not been established. If the prescriber elects to treat such patients with ABILIFY, vigitance should be exercised, particularly for the emergence of difficulty swallowing or excessive somnolence, which could predispose to accidental injury or aspiration. Clinical experience with ABILIIFY in patients with certain concomitant systemic illnesses (see CLINICAL PHARMA-
CoLOGY: Special Populations: Renal Impairment and Hepatic Impairment) is limited. ABILIFY has not been evaluated or used to any appreciable extent in patients with a recent history of myocardial infarction or unstable heart disease. Information for Patients: Physicians are advised to consult full prescribing information to review issues

Drug-Drug Interactions: Given the primary CNS effects of aripiprazole, caution should be used when ABILIFY is taken in combination with other centrally acting
drugs and alcohol. Due to its $\alpha_{1}$-adrenergic receptor antagonism, aripiprazole has the potential to enhance the effect of certain antihypertensive agents. Potential tor Other Drugs to Affect ABILIFY: Aripiprazole is not a substrate of enzymes Aripiprazole also does not undergo direct glucuronidation. This sug-
gests that an interaction of aripiprazole with inhibitors or inducers of these are responsible for aripiprazole metabolism. Agents that induce CYP3A4 (e.g., carbamazepine) could cause an increase in aripiprazole clearance and quinidine, fluoxetine, or paroxetine) can inhibit aripiprazole elimination and (200 mg/day for 14 days) with a $15-\mathrm{mg}$ single dose of aripiprazole increased the effect of a higher ketoconazole dose ( $400 \mathrm{mg} / \mathrm{day}$ ) has not been studied. When concomitant administration of ketoconazole with aripiprazole occurs, aripiprazole dose should be reduced to one-half of its normal dose. Other strong
inhibitors of CYP3A4 (itraconazole) would be expected to have similar effects and inhibitors of CYP3A4 (itraconazole) would be expected to have similar effects and
need similar dose reductions; weaker inhibitors (erythromycin, grapefruit juice)
have not been studied. When the CYP3A4 inhibitor is withdrawn from the combination therapy, aripiprazole dose should then be increased. Quinidine: Coadministration of a $10-\mathrm{mg}$ single dose of aripiprazole with quinidine
$(166 \mathrm{mg} /$ day for 13 days), a potent inhibitor of CYP2D6, increased the AUC of aripiprazole by $112 \%$ but decreased the AUC of its active metabolite, dehydro-
aripiprazole, by $35 \%$. Aripiprazole dose should be reduced to one-half of its noraripiprazole, by $35 \%$. Aripiprazole dose should be reduced to one-half of its normal dose when concomitant administration of quinidine with aripiprazole occur's.
Other significant inhibitors of CYP2D6, such as fluoxetine or paroxetine, would be expected to have similar effects and, therefore, should be accompanied by
similar dose reductions. When the CYP2D6 inhibitor is withdrawn from the combination therapy, aripiprazole dose should then be increased. Carbamazepine: Coadministration of carbamazepine (200 mg BID), a potent CYP3A4 inducer, with
aripiprazole ( $30 \mathrm{mg} Q 0$ ) resulted in an approximate $70 \%$ decrease in $\mathrm{C}_{\text {max }}$ and AUC values of both aripiprazole and its active metabolite, dehydro-aripiprazole. be doubled. Additional dose increases should be based on clinical evaluation. When carbamazepine is withdrawn from the combination therapy, aripiprazole
dose should then be reduced. No clinically significant effect of famotidine, valproate, or lithium was seen on the pharmacokinetics of aripiprazole (see CLINIOther Drugs: Aripiprazole is unlikely to cause clinically important pharmacokinetic interactions with drugs metabolized by cytochrome P450 enzymes. In in
vivo studies, 10 - to 30 -mg/day doses of aripiprazole had no significant effect on metabolism by CYP2D6 (dextromethorphan), CYP2C9 (warrarin), CYP2C19
(omeprazole, warfarin), and CYP3A4 (dextromethorphan) substrates. Additionally, aripiprazole and dehydro-aripiprazole did not show potential for altering
CYP1A2-mediated metabolism in vitro (see CLINICAL PHARMACOLOGY: DrugDrug interactions). Aicohol: There was no significant difference between aripiprazole coadministered with ethanol and placebo coadministered with
ethanol on performance of gross motor skills or stimulus response in healthy subjects. As with most psychoactive medications, patients should be advised to avoid alcohol while taking ABLLIFY. Carcinogenesis,

Pregnancy Category C: There are no adequate and well-controlled studies in pregnant women. It is not known whether aripiprazole can cause fetal harm
when administered to a pregnant woman or can affect reproductive capacity. when administered to a pregnant woman or can affect reproductive capacity.
Aripiprazole should be used during pregnancy only if the potential benefit outweighs the potential risk to the fetus. Labor and Delivery: The effect of
aripiprazole on labor and delivery in humans is unknown. Nursing Mothers: aripiprazole on labor and delivery in humans is unknown. Nursing Mothers: aripiprazole or its metabolites are excreted in human $m$

Pediatric Use: Safety and effectiveness in pediatric and adolescent patients have not been established. Geriatric Use: 0f the 5592 patients treated with
aripiprazole in premarketing clinical trials, $659(12 \%)$ were $\geq 65$ years old and arpiprazole in premarketing clinical trials, $659(12 \%)$ were $\geq 65$ years old and
$525(9 \%)$ were $\geq 75$ years old. The majority $(91 \%)$ of the 659 patients were diagnosed with dementia of the Alzheimer's type. Placebo-controlled studies of aripiprazole in schizophrenia did not include sufficient numbers of subjects aged 65
and over to determine whether they respond differently from younger subjects. There was no effect of age on the pharmacokinetics of a single 15 -mg dose of aripiprazole. Aripiprazole clearance was decreased by $20 \%$ in elderly subjects
( 265 years) compared to younger adult subjects ( 18 to 64 years), but there was no detectable effect of age in the population pharmacokinetic analysis in schizAlzheimer's disease, have suggested that there may be a different tolerability profile in this population compared to younger patients with schizophrenia (see
PRECAUTIONS: Use in Patients with Concomitant Illness). The safety and efficaCy of ABILIFY in the treatment of patlents with psychosis associated with
Alzheimer's disease has not been established. If the prescriber efects to treat Alzheimer's disease has not been established. If the press
such patients with ABILIF, vigilance should be exercised.

\section{ADVERSE REAGTIONS}

Aripiprazole has been evaluated for safety in 5592 patients who participated in multiple-dose premarketing trials in schizophrenia, bipolar mania, and dementia Patients with Schizophrenia The following findings are based on a pool of five placebo-controlled trials (four 4-week and one 6-week) in which aripiprazole Associated with Discontinuation of Treatment in Short-Term, Placebo-Controfled Trials: Overall, there was no difference in the incidence of discontinuation due to adverse events between aripiprazole-treated $(7 \%)$ and placebo-treated $(9 \%)$
patients. The types of adverse events that led to discontinuation were similar between the aripiprazole and placebo-treated patients. Adverse Events
Occurring at an incidence of $>2 \%$ Among Aripiprazole-Treated Patients and Greater than Placebo in Short-Term, Placebo-Controlled Trials: Treatment-emergent adverse events that occurred during acute therapy (up to 6 weeks) at an
incidence of $2 \%$ or more of patients treated with aripiprazole (doses $\geq 2 \mathrm{mg} /$ day) incidence of $2 \%$ or more of patients treated with aripiprazole (doses $\geq 2 \mathrm{mg} /$ day)
and for which the incidence was greater than the incidence reported for placebo were: Body as a Whole - headache, asthenia, and fever; Digestive Systemheadedness, somnolence, akathisia, and tremor; Respiratory System - rhinitis
Dose-Related Adverse Events: The only adverse event to have a possible dose response relationship, and then most prominent only with $30 \mathrm{mg}$, was Extrapyramidal Symptoms: In short-term, placebo-controlled trials, the incidence objectively collected data from those trials on the Simpson Angus Rating Scale nvoluntary Movement Scales (for dyskinesias) also did not show a difference between aripiprazole and placebo, with the exception of the Barnes Akathisia between group comparison for 4 - to 6 -week placebo-controlled trials revealed the proportions of patients experiencing potentially clinically significant changes routine serum chemistry, hematologyy, or urinalysis parameters. Weight Gain. short-term trials, there was a slight difference in mean weight gain between a difference in the proportion of patients meeting a weight gain criterion of $\geq 7 \%$ of body weight [aripiprazole (8\%) compared to placebo $(3 \%)]$. ECG Changes. significant differences between aripiprazote and placebo in the proportion of patients experiencing potentially important changes in ECG parameters; within $Q T_{6}$ interval. Aripiprazole was associated with a median increase in heart rate of 4 beats per minute compared to a 1 beat per minute increase among placebo patients. Other Adverse Events Observed During Clinical Trials: Following is a list reported by patients treated with aripiprazole at multiple doses $\geq 2 \mathrm{mg} / \mathrm{day}$ during any phase of a trial within the database of 5592 patients. It is important to
emphasize that, although the events reported occurred during treatment with aripiprazole, they were not necessarily caused by it. Frequent events occurred in are events in fewer than $1 / 1000$ patients. Body as a Whole. Frequent - flu syndrome, peripheral edema, chest pain, neck pain, neck rigidity; infrequent-pelvic pain, suicide attempt, face edema, malaise, photosensitivity, arm rigidity, jaw pain, chills, bloating, jaw tightness, enlarged abdomen, chest tightness; Rare -
throat pain, back tightness, head heaviness, moniliasis, throat tightness, leg throat pain, back tightness, head heaviness, moniliasis, throat tightness, leg
rigidity, neck tightness, Mendelson's syndrome, heat stroke. Cardiovascular System: Frequent - hypertension, tachycardia, hypotension, bradycardia; val, cardiac arrest, atrial fibrillation, heart failure, AV block, myocardial ischemia, phlebitis, deep vein thrombosis, angina pectoris, extrasystoles; Rare - vasovagal Frequent - anorexia, nausea and vomiting; Infrequent - increased appetite, gas-
troenteritis, dysphagia, flatulence, gastritis, tooth caries, gingivitis, hemorrhoids, gastroesophageal reflux, gastrointestinal hemorrhage, periodontal abscess, tongue edema, fecal incontinence, colitis, rectal hemorrhage, stomatitis, mouth ulcer, cholecystitis, fecal impaction, oral moniliasis, cholelithiasis, eructation,
intestinal obstruction, peptic ulcer; Rare - esophagitis, gum hemorrhage, glossitis, hematemesis, melena, duodenal ulcer, cheilitis, hepatitis, hepatomegaly, pancreatitis, intestinal perforation. Endocrine System: Infrequent - hypothysis, lymphadenopathy, thrombocytopenia; Rare - eosinophilia, thrombocythemia, creatine phosphokinase increased; Infrequent - dehydration, edema, hypercholesteremia, hyperglycemia, hypokalemia, diabetes mellitus, SGPT increased,
hyperlipemia, hypoglycemia, thirst, BUN increased, hyponatremia, SGOT hyperlipemia, hypoglycemia, thirst, BUN increased, hyponatremia, SGOT increased, bilirubinemia, lactic dehydrogenase increased, obesity; Rare - hyperkalemia, gout, hypernatremia, cyanosis, hyperuricemia, hypoglycemic reaction. pain, myasthenia, arthritis, arthrosis, muscle weakness, spasm, bursilis; RareNervous System: Frequent - depression, nervousness, increased salivation, rigidity; Infrequent - dystonia, witch, impaired concentration, paresthesia, vasodilation, hypesthesia, extremity tremor, impotence, bradykinesia, decreased libido, panic attack, apathy, dyskinesia, hypersomnia, vertigo, dysarthria, tardive dyskinesia, ataxia, impaired memory, stupor, increased libido, amnesia, g, myoclonus, dysphoria neuropathy, increased reflexes, slowed thinking, hyperkinesia, hyperesthesia, hypotonia, oculogyric crisis; Rare - delirium,
euphoria, buccoglossal syndrome, akinesia, blunted affect, decreased consciousness, incoordination, cerebral ischemia, decreased reflexes, obsessive pneumonia; Infrequent - asthma, epistaxis, hiccup, laryngitis; Rare - hemoptysis, aspiration pneumonia, increased sputum, dry nasal passages, pulmonary Appendages: Frequent - dry skin, pruritus, sweating, skin ulcer; infrequent maculopapular rash, exfoliative dermatitis, urticaria. Special Senses media, cataract, altered taste, blepharitis; Rare - increased lacrimation, frequent blinking, otitis externa, amblyopia, deafness, diplopia, eye hemorrhage, photophobia. Urogenital System: Frequent - urinary incontinence; infrequentamenorrhea, abnormal ejaculation, vaginal hemorrhage, vaginal moniliasis, kidney failure, uterus hemorrhage, menorrhagia, albuminuria, kidney calculus, noc-
turia, polyuria, urinary urgency; Rare - breast pain, cervicitis, female lactation, anorgasmy, urinary burning, glycosuria, gynecomastia, urolithiasis, priapism.

\section{OVERDOSAGE}

Management of Overdosage: No specific information is available on the treatment of overdose with aripiprazole. An electrocardiogram should be obtained in
case of overdosage and, if $Q T_{C}$ interval prolongation is present, cardiac monitoring should be instituted. Otherwise, management of overdose should concentrate on supportive therapy, maintaining an adequate airway, oxygenation and itoring should continue until the patient recovers. Charcoal - In the event of an overdose of ABILIFY, an early charcoal administration may be useful in partially preventing the absorption of aripiprazole. Administration of $50 \mathrm{~g}$ of activated
charcoal, one hour after a single $15 \mathrm{mg}$ oral dose of aripiprazole, decreased the mean AUC and $\mathrm{C}_{\max }$ of aripiprazole

Controlled Substance: ABILIFY (aripiprazole) is not a controlled substance. Controlled Substance: ABILIFY (aripiprazole) is not a controlled substance.
Abuse and Dependence: Aripiprazole has not been systematically studied in Abuse and Dependence: Aripiprazole has not been systematically studied in
humans for its potential for abuse, tolerance, or physical dependence. In physical dependence studies in monkeys, withdrawal symptoms were observed upon abrupt cessation of dosing. While the clinical trials did not reveal any tendency for possible to predict on the basis of this limited experience the extent to which a CNS-active drug will be misused, diverted, and/or abused once marketed. Consequently, patients should be evaluated carefully for a history of drug abuse,
and such patients should be observed closely for signs of ABLLIFY misuse or
abuse (e.g., development of tolerance, increases in dose, drug-seeking behavior).

Marketed by Otsuka America Pharmaceutical, Inc, Rockville, MD 20850 USA and Bristol-Myers Squibb Co, Princeton, NJ O8543 USA

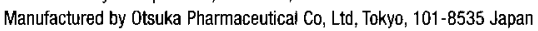
Distributed by Bristol-Myers Squibb Co, Princeton, NJ 08543 USA

Bristol-Myers Squibb Company
Princeton, Nl 08543 U.S.A.

D6-B001A-11-02 A4115/10-02 Issued: November 2002

C2003 Otsuka Pharmaceutical Co, Ltd, Tokyo, 101-8535 Japan 